\title{
Effect of Resuscitation Fluids on the Expression of hsp90a in Cardiac Muscles of Hemorrhagic Shocked Rats
}

\author{
Xiao-Kun Yang ${ }^{1}$, Jie $\mathrm{Su}^{2}$ and Gui-Sen $\mathrm{Xu}^{{ }^{1^{*}}}$ \\ ${ }^{1}$ Department of Emergency, General Hospital of Chengdu Military Command Area, Chengdu 610083, ${ }^{2}$ Department of \\ Emergency, Chengdu First People's Hospital, Chengdu 610016, Sichuan Province, PR China
}

*For correspondence: Email: guisenxucdzy@126.com; Tel: +86 028-86570325; Fax: +86 028-86570325

\begin{abstract}
Purpose: To investigate the protective effects of various resuscitating fluids on severe hemorrhagic shocked (HS) rats by comparing the expression changes of hsp90a in cardiac muscles and survival of rats.

Methods: Western-blot and immunohistochemistry methods were performed to determine hsp90a expressions in cardiac muscles of HS rats following treatment with different resuscitation fluids. In addition, the survival rates of of all the test groups were investigated.

Results: The expression of hsp90a decreased following treatment with resuscitation fluids based on both Western blot and immunohistochemistry data. Ringer lactate solution (RLS) was the most effective therapeutic fluid in this regard, and this was buttressed by survival rate data $(90 \%)$.

Conclusion: The expression of hsp90a in rat cardiac muscle is decreased to various degrees by treatment with different resuscitating fluids, with the effect most pronounced for ringer lactate solution. Furthermore, hsp90a plays an important role in hemorrhagic rat cardiac muscle as a regulatory factor.
\end{abstract}

Keywords: Resuscitating fluids, Hemorrhagic shock, Heat shock protein 90a, hsp90a

Tropical Journal of Pharmaceutical Research is indexed by Science Citation Index (SciSearch), Scopus, International Pharmaceutical Abstract, Chemical Abstracts, Embase, Index Copernicus, EBSCO, African Index Medicus, JournalSeek, Journal Citation Reports/Science Edition, Directory of Open Access Journals (DOAJ), African Journal Online, Bioline International, Open-J-Gate and Pharmacy Abstracts

\section{INTRODUCTION}

Fluid resuscitation is one of the most important methods for early treatment of shock, and effective fluid resuscitation may increase the long-term survival of patients after hemorrhagic shock (HS) [1-4]. Currently, the fluids used for resuscitation mainly include crystalloids and colloids [1]; the common form of crystalloids is the ringer lactate solution, and blood plasma and the blood substitute is the main form of colloids; in addition, hypertonic sodium chloride dextran was reported to have good treatment effects for recovery of the blood volume after HS $[5,6]$.
Secondary multiple organ failure (MOF) may be induced after severe HS. Heart is one of the target organs susceptible to damage by HS, and the change in heart function can affect the haemodynamics and functions of other vital organs, leading to irreversible damage. Therefore, how to protect cardiac function in HS or alleviat dysfunction of cardiac function caused by $\mathrm{HS}$ is a serious problem.

The molecular chaperone, heat shock protein (hsp90), is a universally expressed and abundant 
molecular chaperone, and mammalian hsp90a and hsp90 $\beta$ are two major cellular proteins of the hsp90 subfamily and are highly homologous $(86 \%)$ to each other [7-9]. In our previous study [10], we found that hsp90a can indirectly participate in the protection of myocardial tissue together with other molecules (including IF-1a and HIF-1 $\alpha$ ). Therefore, the present investigation is designed to evaluate the protective effects of some resuscitation fluids on myocardial tissue of HS rats by comparing the expression changes of hsp90a in cardiac muscles and survival of the rats.

Ringer lactate solution (RLS) and hypertonic sodium chloride dextran (HSD) were purchased from Sichuan Kelun Pharmaceutical Co. LTD. (Sichuan, China); Polygeline (PG) was obtained from Wuhan Hualong Bio-chemical Pharmaceutical Co. LTD. (Wuhan, China); Goat anti-rat hsp90a polyclonal IgG was purchased from Merck Drug \& Biotechnology (Germany); Rabbit anti-goat IgG/HRP was purchased from Boster Bio-engineering LTD. (Wuhan, China); nitrocellulose filters were purchased from Beijing Dingguo Biotechnology Co.(Beijing, China); Coomassie Protein Assay kit was purchased from Nanjing Jiancheng Bioengineering Institute (Nanjing, China); immunohistochemistry kit was purchased from Beijing Zhongshan Co. (Beijing, China). All other chemicals used in this study were of analytical reagent grade.

\section{Grouping of animals and protocols}

A total of 240 SD rats were equally divided into the following 4 groups $(n=60)$ : Control group, RLS group, PG group, and HSD group. All the rats were fasted and had free access to water before the experiment. Cannulations were performed in the right femoral artery, right femoral vein and right carotid artery. In addition, a three limb tube, connecting the arterial cannulation, was used to observe blood pressure, exsanguinate, and administer heparin sodium $(500 \mathrm{U} / \mathrm{kg})$ and test resuscitation fluids. The shock model was established by exsanguinating $45 \%$ of the blood of the rats. For the control group, the same volume of physiological saline as blood loss was administered; for the RLS group, the volume of fluid replacement and blood loss were the same; for the PG group, the volume of fluid replacement was $20 \mathrm{ml} / \mathrm{kg}$; for the HSD group, the volume was $6 \mathrm{ml} / \mathrm{kg}$ [12]. Six anesthetized rats were sacrificed to obtain cardiac muscles at 1, 3, 6, 12 and $24 \mathrm{~h}$ after treatment with the various resuscitation fluids. For survival analysis, the remaining 30 animals of each group were freed from the cannula, the opening sutured, and observed for $24 \mathrm{~h}$.

\section{Western blotting}

Total proteins of cardiac muscles were extracted, and then equal amounts of protein $(40 \mu \mathrm{g})$ were separated by sodium dodecyl sulfate/ polyacrylamide gel electrophoresis (SDS/PAGE), blotted on nitrocellulose filters (NCF), and probed with Goat anti-rat hsp90a polyclonal IgG and anti-c-Jun rabbit polyclonal IgG, and subsequently with Rabbit anti-goat IgG/HRP, and detected by chemiluminescence. To measure protein loading, antibody directed against $\beta$-actin was used.

\section{Immunohistochemistry test}

The immunohistochemistry assay was performed following the method described on the commercial kits to examine the expressions of hsp90a in the cardiac muscles.

\section{Statistical analysis}

All the experiments were conducted at least in triplicate and the data are presented as mean \pm SEM. Chi-square of exact test was used to analyze the significance of mouse mortality differences among groups. All other data were evaluated with one-way ANOVA followed by Dunnett multiple comparison test between different groups. Statistical significance of differences was analyzed using SPSS software (SPSS for Windows 15.0, SPSS Inc, USA) at a significance level of $p<0.05$.

\section{RESULTS}

\section{Expressions of hsp90a}

The expressions of hsp90a proteins were determined by western blotting. The expressions of hsp90a proteins (obtained by Western blot) increased after hemorrhagic shock; however, the expressions of hsp90a proteins decreased progressively with the treatment of fluid resuscitation; in addition, it increased rapidly at the 24h after HS (Figs 1 and 2). Furthermore, the expressions of hsp90a protein in cardiac muscles of RLS group were the lowest among all the test groups, and this was followed by the PG group, HSD group, and control group (Fig 2) $(p<$ $0.05)$. The relative protein level was normalized to the Western blot intensity of $\beta$-actin. 


\section{Expression of hsp90a proteins in cardiac muscles}

The expression of hsp90a increased after HS. As can be seen in Fig 3, the expression of hsp90a protein in the cardiac muscles of RLS group was the lowest among all the test groups, followed by PG group, HSD group.

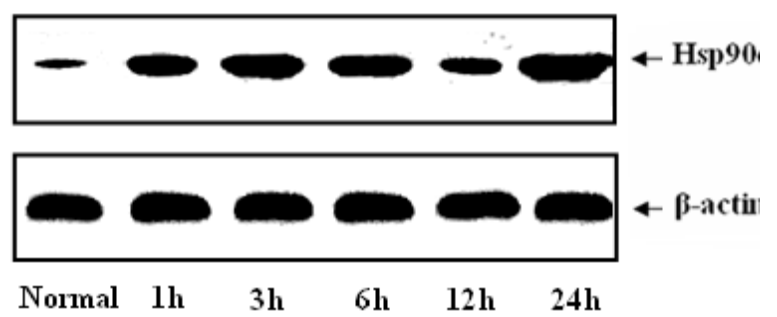

Figure 1: Changes in expression of hsp90a proteins of cardiac muscles after HS

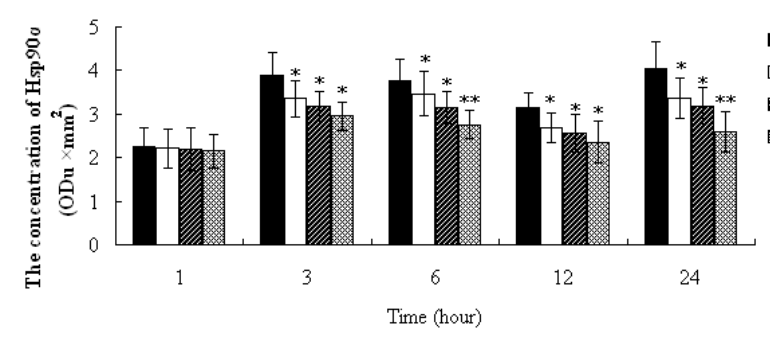

Figure 2: The expressions of hsp90a proteins in cardiac muscles of 4 test groups at different time intervals. (ODu $\times \mathrm{mm}^{2}$, mean $\left.\pm \mathrm{SEM}, \mathrm{n}=6\right)$. Differences amongst the test groups were significant at $3,6,12$ and $24 \mathrm{~h}$ after $\mathrm{HS}(p<0.05)$, compared with the control group.

\section{Survival rate}

As can be seen from Table 1, survival increased significantly following treatment with fluid resuscitation, and this was most pronounced for those administered with RLS (90\%, $p<0.01)$, compared with the control group.
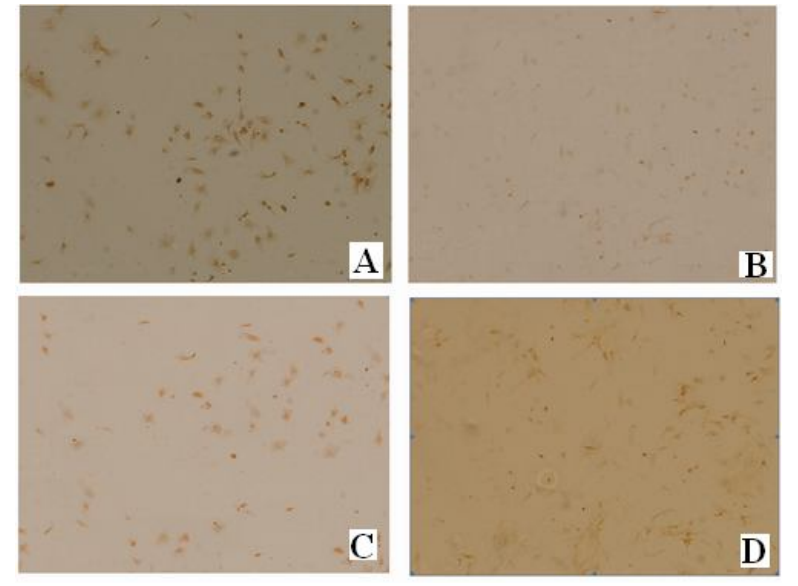

Figure 3: Results of immunohistochemistry test Note: $A=$ Control group; $B=$ RLS group; $C=P G$ group; $D=$ HSD group

\section{DISCUSSION}

i HS can induce a high mortality and morbidity because of hypoperfusion and tissue hypoxia, which tend to induce multiple-organ failure (especially the cardiovascular organs) [13-15]. Heat shock protein Hsp90a is a ubiquitously expressed molecular chaperone, which is necessary for the maintenance of eukaryotic homeostasis. Hsp90a can also be secreted extracellularly and is associated with several physiological and pathological processes including shock, infectious diseases, wound healing, cancer and diabetes [9]. In addition, hsp90a proteins play very important roles in protecting the body from damage resulting from shock, hyperpyrexia, anoxia and infection [16]. In our previous study, we found that hsp90a protein plays an important protective effect on cardiac muscles in the early phase of shock. In addition, the protective effect of hsp90a protein on cardiac muscles is mainly based on its effect as a molecular chaperone by combining with the bHLH-PAS domain of hypoxia inducible factor $1 \alpha$ (HIF-1a) [11].

Table 1: Survival rate of the animals

\begin{tabular}{cccc}
\hline \multirow{2}{*}{ Group } & \multicolumn{3}{c}{ Outcome of intervention } \\
\cline { 2 - 4 } & Death & Survival (N) & Survival (\%) \\
\hline Control & 22 & 8 & 26.67 \\
HSD & 12 & 18 & 60.00 \\
PG & 7 & 23 & 76.67 \\
RLS & 3 & 27 & 90.00 \\
\hline
\end{tabular}

Significant differences were observed between the groups using $X^{2}$ analysis $(p<0.01)$. 
In this study, we investigated the effects of different resuscitation fluids on the expression of hsp90a in HS rats' cardiac muscles, so as to find a safe and effective therapeutic method to treat HS. From the results of our present study, we can come to the conclusion that the hsp90a expressions can be affected significantly by treating with different resuscitation fluids after HS. In addition, the expressions and lasting time of hsp90a in RLS groups is the lowest among all the tested groups, and this is followed by $P G$ group, HSD group. These results indicate that RLS is the most effective resuscitation fluid for the treatment of $\mathrm{HS}$, and also demonstrate that RLS has a very potent protective effect against mortality induced by HS in rats.

\section{CONCLUSION}

In conclusion, the results obtained in this work are noteworthy, and our results demonstrate that resuscitation fluids have favorable protective effects on cardiac muscles of rats with HS, especially RLS, and we suggest that the RLS can be utilized as an effective and safe disease preventive or therapeutic agent for the treatment of HS. However, more investigations are necessary to fully elucidate the mechanism of action of the different resuscitation fluids in the future.

\section{ACKNOWLEDGEMENT}

This work was supported by the Sichuan Provincial Department of Science and Technology (no. 2010JY0186).

\section{REFERENCES}

1. Kentner R, Safar P, Prueckner S, Behringer W, Wu X Henchir J. Titrated hypertonic/hyperoncotic solution for hypotensive fluid resuscitation during uncontrolled hemorrhagic shock in rats. Resuscitation 2005; 65: 87-95.

2. Arlati S, Storti E, Pradella V, Bucci L, Vitolo A, Pulici M. Decreased fluid volume to reduce organ damage: A new approach to burn shock resuscitation? $A$ preliminary study. Resuscitation 2007; 72: 371-378.

3. Nolan J. Fluid resuscitation for the trauma patient. Resuscitation 2001; 48: 57-69.

4. Hachimi-ldrissi S, Yang $X$, Nguyen DN, Huyghens $L$. Combination of therapeutic mild hypothermia and delayed fluid resuscitation improved survival after uncontrolled haemorrhagic shock in mechanically ventilated rats. Resuscitation 2004; 62: 303-310.

5. Doyle JA, Davis DP, Hoyt DR. The use of hypertonic saline in the treatment of trauma brain injury. $J$ Trauma 200l; 50: 367-383.

6. Pfenninger J, Wagner BP. Hypertonic saline in severe pediatric head injury. Crit Can Med 200l; 29: 1489.

7. Zhang SL, Yu J, Cheng XK, Ding L, Heng FY, Wu NH, et al. Regulation of human hsp90K gene expression. FEBS Letters 1999; 444: 130-135.

8. Cooper LC, Prinsloo E, Edkins AL, Blatch GL. Hsp90a/b associates with the GSK3b/axin1/phospho-bcatenin complex in the human MCF-7 epithelial breast cancer model. Biochemical and Biophysical Research Communications 2011; 413: 550-554.

9. Song $X M, L u o ~ Y Z$. The regulatory mechanism of Hsp90a secretion from endothelial cells and its role in angiogenesis during wound healing. Biochemical and Biophysical Research Communications 2010, 398: 111-117.

10. Chen $X Y$, Yang $X K$. Expression and change of hsp90a induced by hypoxia in neonatal rats' cardiac cells. $J$ Lanzhou Univ (Med Sci) 2007, 33: 17-20.

11. National Institute of Health, USA. Public health service policy on humane care and use of laboratory animals; 2002.

12. Li T, Liu LM, Diao YF, Liao ZF, Fan XQ, Chen F. On proper volume of different fluids for hemorrhagic shock resuscitation in rats. Acta Acad Med Milit Tert 2008; 30: 199-202.

13. Suo $X Y, D u Z H$, Wang $H S$, Li JG, Wang YL, Yao SD, Chen WM. The effects of stimulation at acupoint ST36 points against hemorrhagic shock in dogs. Am J Emer Med 2011; 29: 1188-1193.

14. McDonald M, Abdelrahman M, Cuzzocrea S. Tyrphostin reduces the organ injury in haemorrhagic shock: role of inducible nitric oxide synthase. Resuscitation 2003; 58: 349-361.

15. Md S, Moochhala SM, Siew Yang KL, Lu J, Anuar F, Mok P, Ng KC. The Role of Selective Nitric Oxide Synthase Inhibitor on Nitric Oxide and PGE2 Levels in Refractory Hemorrhagic-Shocked Rats. J Surg Res 2005; 123: 206-214.

16. Csermely $P$, Schnaider T, Soti C, Prohaszka Z, Nardai C. The $90-k D a$ molecular chaperone family: structure, function, and clinical applications. Acomprehensive review. Pharmacol Ther 1998; 79: 129-168. 